\title{
Feasibility guidelines for kaonic-atom experiments with ultra-high-resolution X-ray spectrometry
}

\author{
E. Friedman ${ }^{\mathrm{a}, *}$, S. Okada ${ }^{\mathrm{b}}$ \\ ${ }^{a}$ Racah Institute of Physics, The Hebrew University, 91904 Jerusalem, Israel \\ ${ }^{b}$ RIKEN Nishina Center, RIKEN, Wako, 351-0198, Japan
}

\begin{abstract}
Recent studies of strong interaction effects in kaonic atoms suggest that analysing so-called 'lower' and 'upper' levels in the same atom could separate one-nucleon absorption from multinucleon processes. The present work examines the feasibility of direct measurements of upper level widths in addition to lower level widths in future experiments, using superconducting microcalorimeter detectors. About ten elements are identified as possible candidates for such experiments, all of medium-weight and heavy nuclei. New experiments focused on achieving good accuracy for widths of such pairs of levels could contribute significantly to our knowledge of the $K^{-}$-nucleon interaction in the nuclear medium.
\end{abstract}

Keywords:

kaonic atoms, antikaon-nucleon interaction, microcalorimeter

\section{Introduction}

Results of the precision measurements of kaonic hydrogen atoms by the SIDDHARTA collaboration [1, 2] form already part of the data-base used by Ikeda, Hyodo and Weise (IHW) in constructing the antikaon-nucleon scattering amplitudes near threshold [3, 4]. Studies of kaonic atoms using potentials built on sub-threshold in-medium antikaon-nucleon scattering amplitudes [5, 6, 7] clearly indicate that multinucleon processes contribute significantly to the observed strong-interaction level shifts and widths. In

*Corresponding author: E. Friedman, elifried@cc.huji.ac.il 
Table 1: rms radii of various terms of the $\mathrm{K}^{-}$-nucleus potential (in $\mathrm{fm}$ ). $\mathrm{r}_{m}$ is the $\mathrm{rms}$ radius of the nucleus.

\begin{tabular}{cccccccc}
\hline & $\mathrm{r}_{m}$ & $\operatorname{Re}($ full $)$ & $\operatorname{Re}(1 \mathrm{~N})$ & $\operatorname{Re}(\mathrm{mN})$ & $\operatorname{Im}($ full $)$ & $\operatorname{Im}(1 \mathrm{~N})$ & $\operatorname{Im}(\mathrm{mN})$ \\
\hline $\mathrm{Ni}$ & 3.72 & 3.34 & 3.82 & 2.86 & 3.73 & 4.46 & 3.12 \\
$\mathrm{~Pb}$ & 5.56 & 5.21 & 5.71 & 4.78 & 5.46 & 6.23 & 5.00 \\
\hline
\end{tabular}

particular it was shown [8] using the IHW amplitudes that analysing socalled 'lower' and 'upper' levels in the same atom could separate one-nucleon $(1 \mathrm{~N})$ absorption from multinucleon $(\mathrm{mN})$ processes. This property is the result of the very different radial dependences of the $1 \mathrm{~N}$ and $2 \mathrm{~N}$ terms of the potential, as demonstrated for $\mathrm{Ni}$ and $\mathrm{Pb}$ in table 1, It is seen that in both examples the rms radius of the $1 \mathrm{~N}$ real term is larger than that for the $\mathrm{mN}$ real term by $0.95 \mathrm{fm}$ and that for the imaginary part the difference is 1.2 to $1.3 \mathrm{fm}$.

With the one-nucleon amplitudes firmly based on the SIDDHARTA experiment and its subsequent analyses, there is now a possibility to gain information on multinucleon processes of antikaons in nuclei. This calls for reduced uncertainties in experimental results, particularly for the upper level widths.

Strong interaction effects in exotic atoms have been studied in great detail for several decades, see [9] for a recent review. Regarding strengths of absorption, kaonic atoms are intermediate between weak absorption in pionic atoms and very strong absorption in antiprotonic atoms. Absorption is sufficiently strong to make it the dominant effect in kaonic atoms, where strong-interaction level widths are up to one order of magnitude larger than the corresponding strong-interaction level shifts. Furthermore, these shifts are almost universally repulsive, although the real potentials required to fit kaonic atom data are attractive. Hence the role of the real part is secondary to that of the imaginary part of the potential.

The level width which is usually obtained as the imaginary part of the complex eigenvalue when solving the Klein-Gordon equation with an optical potential [10] is also related to the imaginary part of the potential as follows

$$
\Gamma_{\mathrm{st}}=-2 \frac{\int \operatorname{Im} V_{\mathrm{opt}}|\psi|^{2} d \vec{r}}{\int\left[1-\left(B+V_{\mathrm{C}}\right) / \mu\right]|\psi|^{2} d \vec{r}}
$$


where $B, V_{\mathrm{C}}$ and $\mu$ are the $K^{-}$binding energy, Coulomb potential and reduced mass, respectively. (For a Schroedinger equation the denominator is just the normalizing integral.) The widths are therefore the quantities which are more directly connected to the potential.

All available experimental results regarding upper levels in kaonic atoms are in the form of relative yields, defined as

$$
Y^{\mathrm{rel}}=\Gamma_{\mathrm{rad}} /\left(\Gamma_{\mathrm{rad}}+\Gamma_{\mathrm{st}}\right)
$$

where $\Gamma_{\text {rad }}$ and $\Gamma_{\text {st }}$ are the radiation width of the upper to lower level transition and the strong interaction width of the upper level, respectively. The relative error of the derived $\Gamma_{\text {st }}$ which is the quantity of interest turns out to be the relative error of the measured yield divided by $\left(1-Y^{\mathrm{rel}}\right)$, introducing conflicting demands between intensity and accuracy.

The available data for kaonic atoms are based on experiments of three to four decades ago [11]. Although the data cover the whole of the periodic table with reasonably good accuracy for the shifts and widths of the lower levels, the widths of the upper levels are determined from the measured relative yields of the upper to lower level transitions. This causes an increase of errors on top of the usually poorer accuracies in the determination of yields. It is therefore desirable to carry out new experiments on several carefully selected targets where improved accuracy in determining strong-interaction observable for both lower and upper levels in the same atom is the prime concern. Recent development of a high resolution gamma-ray spectrometer based on superconducting microcalorimeters makes it possible, for the first time, to consider direct measurements of widths of upper levels in kaonic atoms.

Section 2 outlines basic features of such high-resolution detector and section 3 explains the method used in the present survey, looking for suitable targets for future kaonic atom experiments. All calculations are based as much as possible on information gained from global fits to existing data from kaonic atoms. The results of the survey are presented in section 4. These include widths of the various levels involved and values of $\mathrm{E}_{\mathrm{X}}$, the energy of the X-ray transition feeding into the upper level. Relative and absolute yields of relevant transitions are also shown, to serve as guidance for further detailed planning of experiments. Section [5 is a discussion and summary, addressing also the question of the ability of restricted data to provide useful information. 


\section{Detectors}

In recent years, cryogenic particle detectors have achieved remarkable development [12]. Above all, superconducting microcalorimeters based on transition-edge sensor (TES) offer unprecedented energy resolution for single photon detections from the near infrared through gamma rays. Thanks to recent technological advances in multiplexed readout of a TES multi-pixel array, the large-area ultra-high-resolution TES detector has now edged closer to practical use in a variety of nuclear measurements.

For gamma-ray spectroscopy, the energy resolution for a single pixel so far achieved is $22-\mathrm{eV}$ resolution (FWHM) at $\sim 100 \mathrm{keV}[13]$, being more than an order of magnitude better than high-purity germanium detectors. An average resolution of $53 \mathrm{eV}$ at $\sim 100 \mathrm{keV}$ was achieved for a 256 pixel spectrometer with a collecting area of $5 \mathrm{~cm}^{2}$ [14]. The useable dynamic range of this detector is above $400 \mathrm{keV}$ and the energy resolution $\Delta E$ is almost independent of the X-ray energy within the linear range of the detector. In general $\Delta E$ is expected to be proportional to the square-root of this upper limit.

In the present work we take the energy resolution of $53 \mathrm{eV}$ at $\sim 100 \mathrm{keV}$ and either keep it fixed for all X-ray energies or scale it for energy $E_{X} \mathrm{keV}$ as $53 \times \sqrt{E_{X} / 100} \mathrm{eV}$. We chose also this scaling to cover possible different optimizations of resolution and linearity, to include also more conservative estimates in the feasibility study.

\section{Method}

In order to base the present study as much as possible on the available kaonic atom data we use phenomenological $K^{-}$-nucleus potentials that produces very good fits to the data [8, 11],

$$
V_{\mathrm{opt}}=-(4 \pi / 2 \mu)\left[b \rho+B \rho\left(\rho / \rho_{0}\right)^{0.25}\right],
$$

where $\mu$ is the kaon-nucleus reduced mass, $b$ and $B$ are energy-independent complex parameters and $\rho$ is the local nuclear density. Parameter values were $b=-0.15+\mathrm{i} 0.62 \mathrm{fm}, B=1.65-\mathrm{i} 0.045 \mathrm{fm}, \rho_{0}=0.17 \mathrm{fm}^{-3}$, producing $\chi^{2}=103$ for 65 data points. This potential was used for calculating binding energies and strong interaction widths for all kaonic atoms concerned. Similar results are obtained also from potentials of ref. [8]. 
Searching for possible candidates for new kaonic atom experiments, we are interested in X-ray transitions between three 'circular' states, i.e. where the radial number $n$ is $l+1$. The lower level $(\mathrm{L})$ is the one where the $\mathrm{X}$ ray cascade terminates due to dominance of the absorption and the upper level (U) has $n$ larger by 1 . If the width of the upper level $\left(\Gamma_{\mathrm{U}}\right)$ is to be measured directly, then the transition from yet another level $(U+1)$ must be considered. The energy of this transition is denoted by $\mathrm{E}_{\mathrm{X}}$. Transitions from larger $n$-values could be useful too.

With the parameters listed above it is possible to calculate strong interaction level shifts and widths as well as upper level relative yields and, of course, also transition energies. However, to complete the picture regarding feasibility of measurements it is necessary to obtain also reliable estimate of absolute yields of the various transitions. That was achieved by performing calculations of the atomic cascade process within the exotic atom using a program due to Batty [15] which was developed from the program of Huefner [16]. Cascade calculations require an initial distribution of the population of high- $n$ states for the various values of the orbital angular momentum $l$. A statistical $(2 l+1)$ distribution was assumed but deviations from it were also considered by multiplying $(2 l+1)$ by $e^{\alpha l}$ for several values of $\alpha$ as was done in other studies of exotic atoms [17].

\section{Results}

We have calculated strong interaction width for the lower level $\left(\Gamma_{\mathrm{L}}\right)$ and for the upper level $\left(\Gamma_{\mathrm{U}}\right)$ as well as the energy $\mathrm{E}_{\mathrm{X}}$ of the transition into the upper level in many kaonic atoms along the periodic table. Relative and absolute yields of the relevant transition were also calculated. In order to assess the feasibility of direct measurements of $\Gamma_{U}$, this width should be compared with the expected resolution of the microcalorimeter detector. Because of possible dependence on the measured energy itself, we show in the following the relative resolution, i.e. $\Gamma_{\mathrm{U}} / \mathrm{E}_{\mathrm{X}}$, the ratio of the width to the transition energy to be measured. When this quantity is of the order of or larger than the corresponding ratio for the detector resolution then the experiment is deemed feasible. The various efficiencies are discussed later.

Starting with light elements, it became clear that the necessary conditions for direct measurement of width of the upper levels in kaonic atoms are hard to meet. Therefore we do not present any results for kaonic atoms with $2 p$ as 

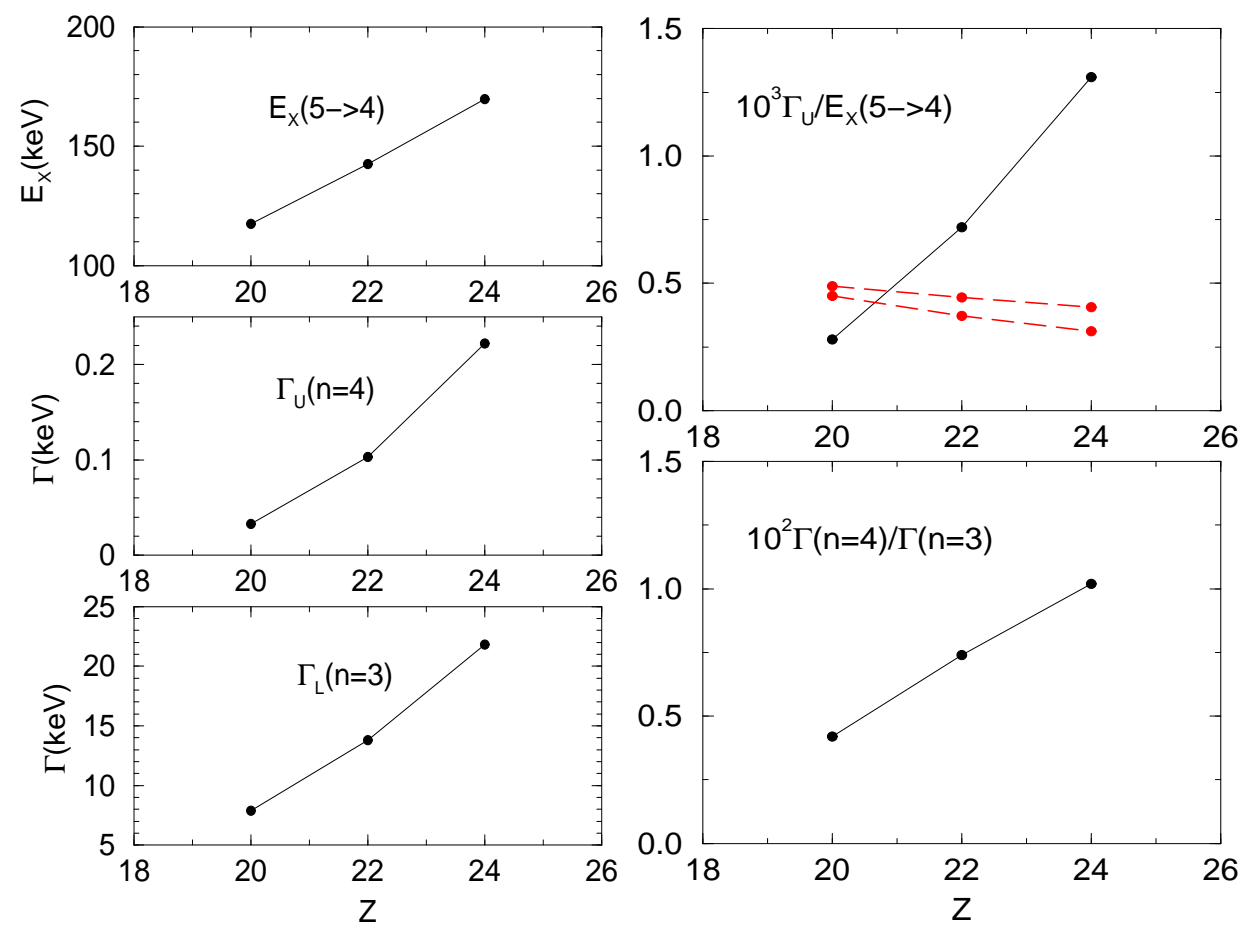

Figure 1: Left: calculated strong-interaction widths of lower $(3 d)$ and upper $(4 f)$ levels and the X-ray energy of the transition feeding into the upper level. Right: ratios of upper to lower level widths $\left(\mathrm{x} 10^{2}\right)$ and ratios of the upper level width to the energy of the transition into that level $\left(\mathrm{x} 10^{3}\right)$. The dashed lines represents estimated lower limits for measuring $\Gamma_{\mathrm{U}}$, see text.

the lower level. This is not a severe restriction considering that our interest in multinucleon absorption processes is aimed at interactions in nuclear matter.

Figure 1 shows results for a $3 d$ lower level in kaonic atoms of $\mathrm{Ca}$, $\mathrm{Ti}$ and Cr. The left-hand side shows widths of the lower and of the upper level and also the energy of the X-ray transition feeding into the upper level. The righthand side of the figure refers to the feasibility of a direct measurement of $\Gamma_{U}$. The lower panel shows the ratios $\Gamma_{\mathrm{U}} / \Gamma_{\mathrm{L}}$ (in units of $10^{-2}$ ), to demonstrate that with a conventional Ge detector which measures $\Gamma_{\mathrm{L}}$ it is usually unfeasible to measure also $\Gamma_{\mathrm{U}}$. The upper panel refers to the direct measurement criterion where $\Gamma_{\mathrm{U}} / \mathrm{E}_{\mathrm{X}}$ (continuous curve) should be comparable or larger than the relative resolution of the detector (dashed curves) in order that the measurement be considered feasible. The lower dashed curve represents a 

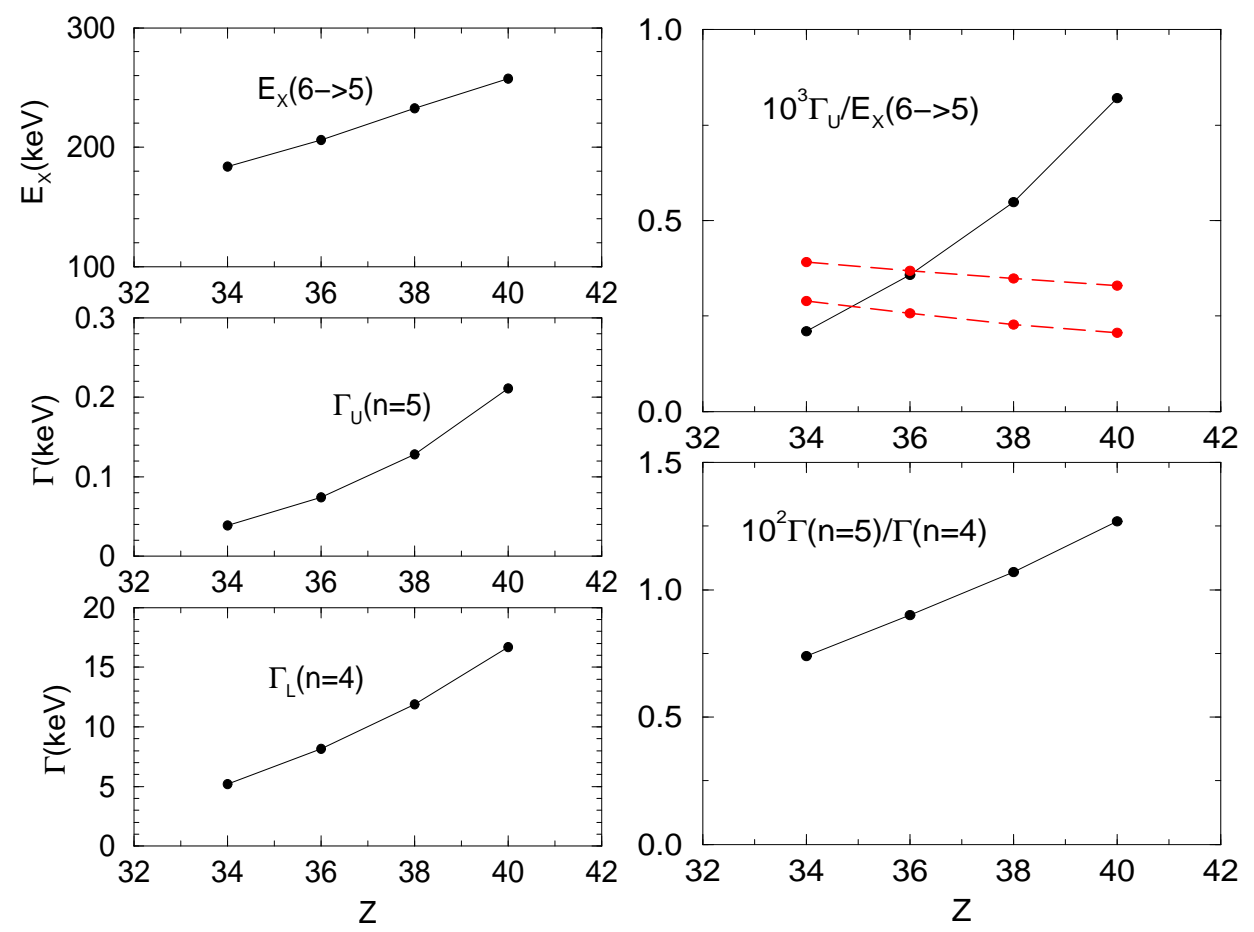

Figure 2: Same as fig 1 but for the $4 f$ and $5 g$ levels.

fixed value of $\Delta E=53 \mathrm{eV}$ between 100 and $400 \mathrm{keV}$ and the upper dashed curve is for $\Delta E=53 \times \sqrt{E_{X} / 100} \mathrm{eV}$ where $E_{X}$ is in $\mathrm{keV}$. It is seen that whereas $\mathrm{Cr}$ and possibly Ti could meet the feasibility criterion, the widths of the the lower levels are likely to be prohibitively large. Note that all of the measured lower level widths so far [11] are well below $10 \mathrm{keV}$. Measuring larger widths could be adversely affected by background. It is evident that there is some conflict between the requirements considering the two widths.

Figure 2 shows similar results for the $4 f$ lower and $5 g$ upper levels. The conflict is here less severe, with $\mathrm{Kr}, \mathrm{Sr}$ and $\mathrm{Zr}$ exceeding the minimum criteria and with the width of the lower level for Sr only slightly exceeding $10 \mathrm{keV}$. Further up the periodic table Fig. 3 shows results for the $5 g$ lower and $6 h$ upper levels and it is seen that if the $\Gamma_{\mathrm{U}} / \mathrm{E}_{\mathrm{X}}$ criterion is relaxed somewhat, then for $Z=54-56$ the lower level width is in the range of previous successful measurements.

Finally, Fig. 4 shows that heavy elements are the most suitable targets 

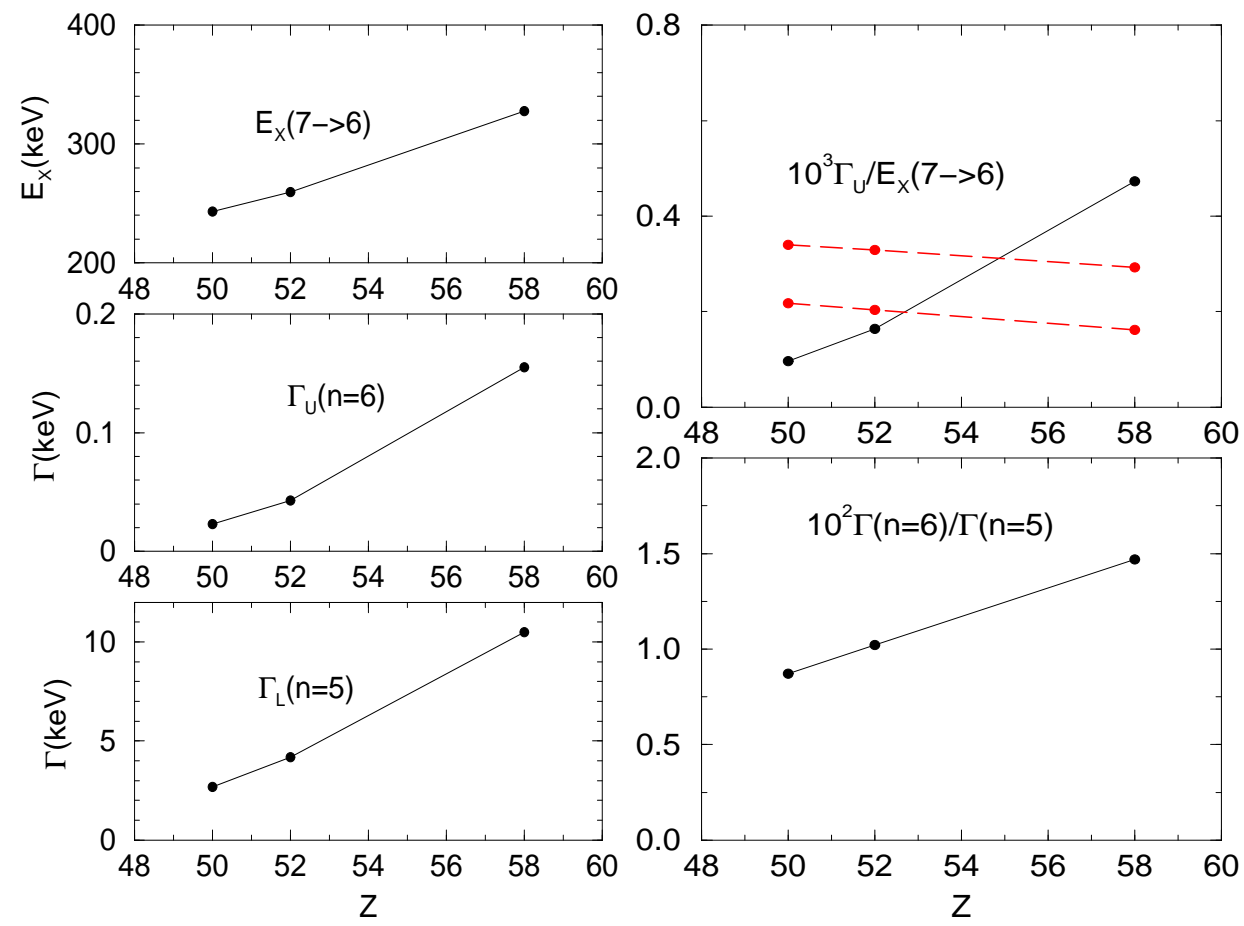

Figure 3: Same as fig 1 but for the $5 g$ and $6 h$ levels.

for the required new kaonic atoms experiments. Note that strong interaction measurements in kaonic atoms of $\mathrm{Yb}, \mathrm{Ta}$ and $\mathrm{Pb}$ have been carried out before, albeit with rather modest accuracies.

Table 2 summarizes values of relative and absolute yields, calculated from the same potential as all other quantities presented above. (The absolute yield is the X-ray yield per stopped kaon). Relative yields are given for the upper to lower level transitions. If upper level widths cannot be determined directly, they can be determined from the measured relative yields, Eq. (22). It is gratifying to note that all the relative yields are smaller than $0.25-0.33$ so that the increase of the relative errors of the corresponding width due to the $1 /\left(1-Y^{\text {rel }}\right)$ factor is not too large.

Strong interaction widths of many other levels are part of the input into the atomic cascade calculations and these were calculated from the same potential Eq. (3). The results in the table were calculated assuming the standard statistical distribution of the population of $l$ values for large $n$. 

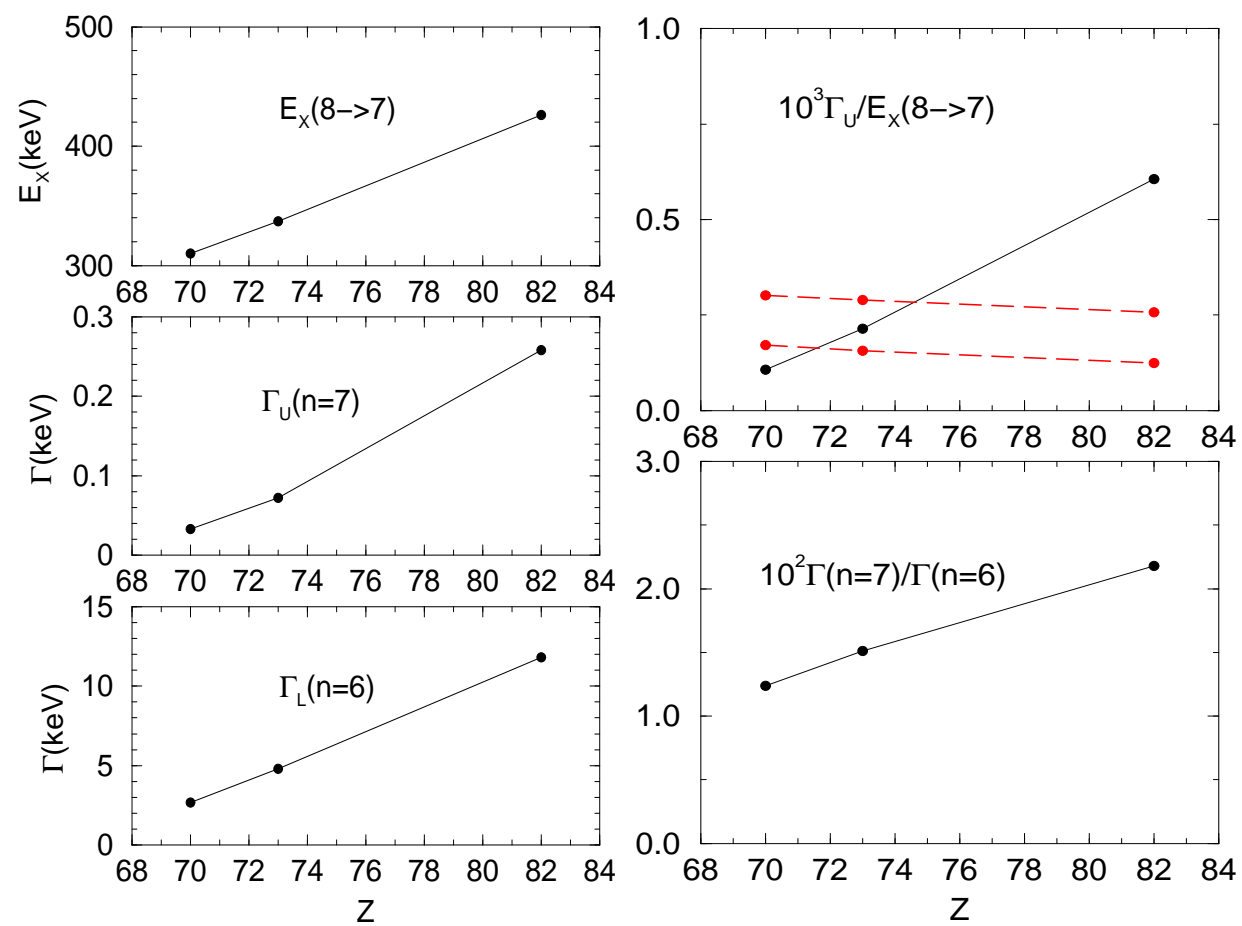

Figure 4: Same as fig 1 but for the $6 h$ and $7 i$ levels.

Varying the parameter $\alpha$ in the range of \pm 0.1 can change absolute yields by up to $\pm 20 \%$. However, absolute yields do not serve any purpose in the analysis; they should be regarded only as estimates that are useful in planning experiments.

Figure 5 shows a summary of the present results for upper levels and the guideline based on the detector resolution. The lower dashed curve represents a fixed value of $\Delta E=53 \mathrm{eV}$ between 100 and $400 \mathrm{keV}$ and the upper dashed curve is for $\Delta E=53 \times \sqrt{E_{X} / 100} \mathrm{eV}$ where $E_{X}$ is in $\mathrm{keV}$. However, the widths of lower levels must also be taken into consideration in planning experiments, as discussed above. Almost all the targets listed here have been used in previous exotic-atom (pionic, kaonic and antiprotonic) experiments [9], many as separated isotopes. 
Table 2: Absolute and relative yields of the relevant transitions for kaonic atoms indicated in the figures. Also listed are the $(n, l)$ values of the various levels, see text.

\begin{tabular}{ccccccc}
\hline target & $\mathrm{U}+1$ & $\mathrm{U}$ & $\mathrm{L}$ & $\mathrm{Y}_{\mathrm{U}+1 \rightarrow \mathrm{U}}^{\mathrm{abs}}$ & $\mathrm{Y}_{\mathrm{U} \rightarrow \mathrm{L}}^{\mathrm{abs}}$ & $\mathrm{Y}_{\mathrm{U} \rightarrow \mathrm{L}}^{\mathrm{rel}}$ \\
\hline $\mathrm{Ca}$ & $(5,4)$ & $(4,3)$ & $(3,2)$ & 0.665 & 0.044 & 0.061 \\
$\mathrm{Ti}$ & $(5,4)$ & $(4,3)$ & $(3,2)$ & 0.627 & 0.019 & 0.029 \\
$\mathrm{Cr}$ & $(5,4)$ & $(4,3)$ & $(3,2)$ & 0.570 & 0.012 & 0.019 \\
& & & & & & \\
$\mathrm{Se}$ & $(6,5)$ & $(5,4)$ & $(4,3)$ & 0.705 & 0.091 & 0.121 \\
$\mathrm{Kr}$ & $(6,5)$ & $(5,4)$ & $(4,3)$ & 0.689 & 0.061 & 0.083 \\
$\mathrm{Sr}$ & $(6,5)$ & $(5,4)$ & $(4,3)$ & 0.663 & 0.043 & 0.062 \\
$\mathrm{Zr}$ & $(6,5)$ & $(5,4)$ & $(4,3)$ & 0.628 & 0.031 & 0.047 \\
& & & & & & \\
$\mathrm{Sn}$ & $(7,6)$ & $(6,5)$ & $(5,4)$ & 0.706 & 0.218 & 0.292 \\
$\mathrm{Te}$ & $(7,6)$ & $(6,5)$ & $(5,4)$ & 0.696 & 0.152 & 0.207 \\
$\mathrm{Ba}$ & $(7,6)$ & $(6,5)$ & $(5,4)$ & 0.651 & 0.069 & 0.101 \\
& & & & & & \\
$\mathrm{Yb}$ & $(8,7)$ & $(7,6)$ & $(6,5)$ & 0.674 & 0.236 & 0.331 \\
$\mathrm{Ta}$ & $(8,7)$ & $(7,6)$ & $(6,5)$ & 0.655 & 0.147 & 0.212 \\
$\mathrm{~Pb}$ & $(8,7)$ & $(7,6)$ & $(6,5)$ & 0.601 & 0.067 & 0.107 \\
\hline & & & & & & \\
& & & &
\end{tabular}




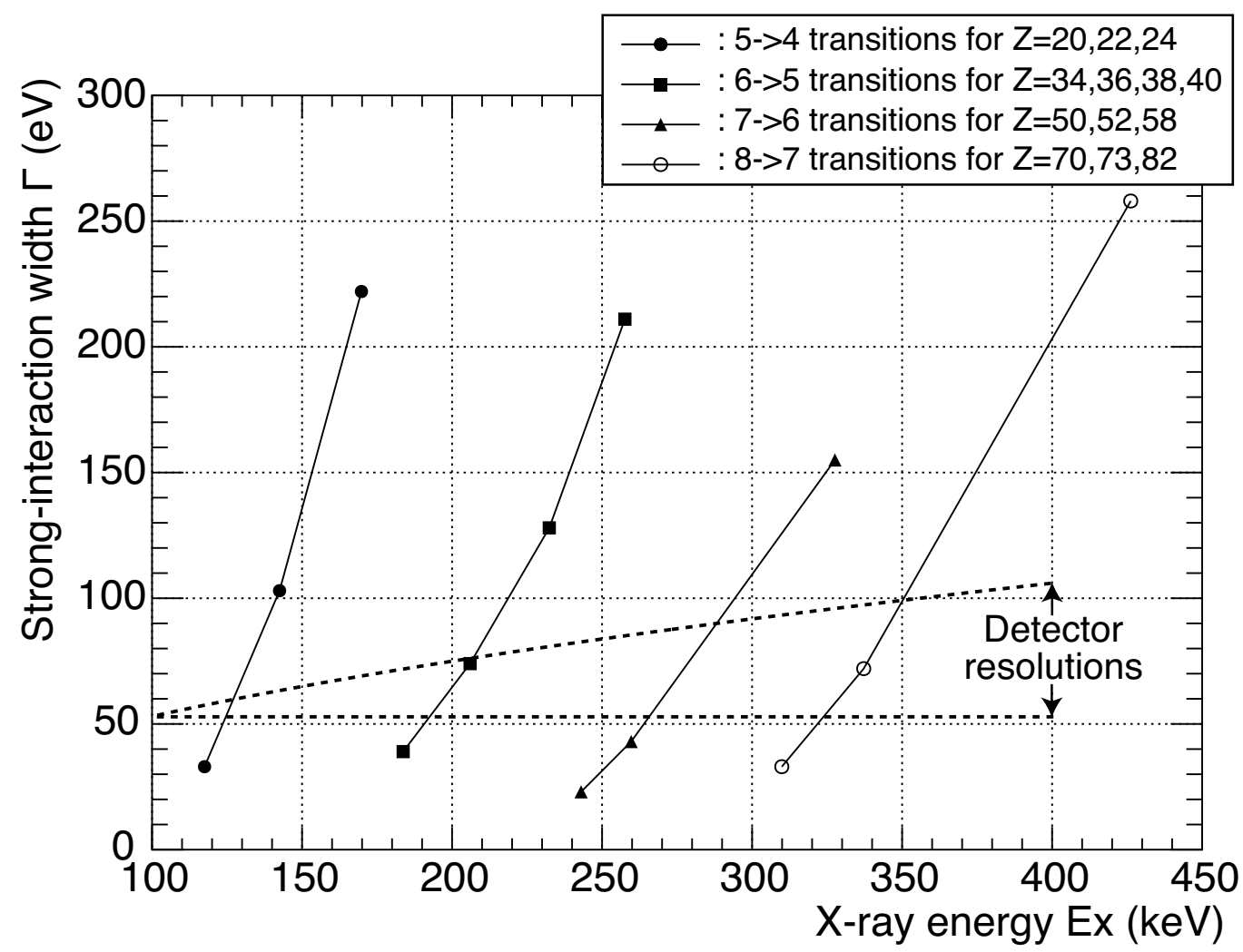

Figure 5: Summary of upper-level results and the feasibility guideline due to the detector resolution.

\section{Discussion and summary}

Along the atomic cascade in exotic atoms the radiation width for the different levels varies rather slowly while the width for absorption by the nucleus varies exponentially, as it depends on the overlap of the atomic wavefunction with the nucleus, Eq. (11). That causes the cascade process to terminate within one or two levels when the nuclear absorption dominates. Direct measurements of strong interaction widths of two levels require the measurement of two successive X-ray transitions. The present results suggest that there could only be a handful of elements where the required conditions are met and it raises the question of whether experiments on a few elements could match the present data base which includes 24 different species of kaonic atoms. 
The question of whether a partial set of data for kaonic atoms could provide similar information to what is obtained from the full data was discussed already in [18], showing that indeed it was possible. Here we have compared the results of global fits to the present-day full data set with results of similar fits to part of the data. Selecting a partial set of the full data, namely $\mathrm{Si}, \mathrm{S}$, $\mathrm{Ni}, \mathrm{Cd}, \mathrm{Sn}, \mathrm{Yb}$ and $\mathrm{Ta}$ that represent the four groups in table 2, we obtain parameter values and uncertainties very close to what is found in fits to the full data. This suggests that a set of about six targets, carefully selected, will be equivalent to the full data base, particularly if the quality of the data is at least as good as that of the old data.

Finally, test calculations show that uncertainties of parameters of the best-fit potentials indeed go down by close to $50 \%$ if upper level widths replace relative yields in the data. We conclude that new kaonic atom experiments on several carefully selected targets using state-of-the-art microcalorimetric spectroscopy could provide widths of lower and upper levels in the same atom which, in turn, could enhance our understanding of multinucleon absorption of antikaons in the nuclear medium.

\section{Acknowledgements}

Discussions with A. Gal and correspondence with W.B. Doriese and J.N. Ullom are gratefully acknowledged. This work was supported by the EU initiative FP7, HadronPhysics3, under the SPHERE and LEANNIS cooperation

programs, by JSPS KAKENHI Grant Number 25105514 and by Incentive Research Grant from RIKEN.

\section{References}

[1] SIDDHARTA collaboration, M. Bazzi, et al., Phys. Lett. B 704 (2011) 113.

[2] SIDDHARTA collaboration, M. Bazzi, et al., Nucl. Phys. A 881 (2012) 88.

[3] Y. Ikeda, T. Hyodo, W. Weise, Phys. Lett. B 706 (2011) 63.

[4] Y. Ikeda, T. Hyodo, W. Weise, Nucl. Phys. A 881 (2012) 98. 
[5] A. Cieplý, E. Friedman, A. Gal, D. Gazda, J. Mareš, Phys. Lett. B 702 (2011) 402.

[6] A. Cieplý, E. Friedman, A. Gal, D. Gazda, J. Mareš, Phys. Rev. C 84 (2011) 045206.

[7] E. Friedman, A. Gal, Nucl. Phys. A 881 (2012) 150.

[8] E. Friedman, A. Gal, Nucl. Phys. A 899 (2013) 60.

[9] E. Friedman, A. Gal, Phys. Rep. 452 (2007) 89.

[10] C.J. Batty, E. Friedman, A. Gal, Phys. Rep. 287 (1997) 385.

[11] see E. Friedman, A Gal, C.J. Batty, Nucl. Phys. A 579 (1994) 518.

[12] C. Enss (ed.), Cryogenic Particle Detection, Topics in Applied Physics, vol. 99, Springer, 2005.

[13] M.K. Bacrania et al., IEEE Trans. Nucl. Sci., 56 (2009) 2299.

[14] D.A. Bennett et al., Rev. Sci. Instrum. 83 (2012) 093113.

[15] C.J. Batty, private communication.

[16] J. Huefner, Z. Phys. 195 (1966) 365.

[17] C.J. Batty, E. Friedman, A. Gal, Phys. Rev. C 59 (1998) 295.

[18] E. Friedman, A. Gal, J. Mareš and A. Cieplý, Phys. Rev. C 60 (1999) 024314 . 